\title{
LISTING OF CORAL CORES AND SLABS AT THE UNITED STATES GEOLOGICAL SURVEY COASTAL CENTER 600 4TH ST. SOUTH ST. PETERSBURG FL. 33701 (813) 893-3684
}

COMPILED BY A. J. JOSEPH, J. H. HUDSON AND R. B. HALLEY $12 / 1 / 1990$

U.S.G.S. OPEN FILE REPORT 91-45 
The 364 cores listed in this catalog were collected by J. Harold Hudson between 1974 and 1990 with the assistance of Dong Ryong Choi, Renato Diaz, Robert Dill, Richard Dodge, Ellen Druffel, Steven Early, Joseph Ghiold, Steven Giddings, Carlos Goenoga, Sheila Griffin, John Halas, Robert Halley, Walter Jaap, Jim Kendall, Jack Kindinger, Lee Kumph, Valerie Paul, Daniel Robbin, Carol Lee Roark, Philip Shea, E. A. Shinn, Pete Spicer, Jack Thompson, Margarite Toscano, and Jenny Wheaton

With few exceptions, cores from the Atlantic are from hemispherical, relatively smooth and non-branching forms of Montastrea annularis. Pacific corals are from similar growth forms of Porites lutea. Exceptions are noted in the listing. All cores have been slabbed (approx. $5 \mathrm{~mm}$ thick) and are accompanied by $\mathrm{x}$-radiograph negatives

Information about the cores is presented as indicated below:

General Location Florida

Place name - Ajax Reef $\left(25^{\circ} 22^{\prime} N ; 80^{\circ} 05 W^{\prime}\right)$ approximate location Time of collection -July, 1984

Coral core number or letter and approximate core length in centimeters \#1(22);

Second core from same coral; \#1'(24);

Core from second coral in immediate area \#2(18)

For information about access to these cores, contact Robert B. Halley at the USGS Coastal Center, 600 4th St. South, St. Petersburg, FL 33701 (813) 8933684 x3020 


\section{Florida Keys}

-Shinn Shoal (250 27.0' $\left.N ; 80^{\circ} 07.3^{\prime} \mathrm{W}\right)$

-March, 1975

-Cores

\#1(33), \#2(33)

\section{-Biscayne National Park}

-July, 1986

-Site $1\left(25^{\circ} 28.9^{\prime} \mathrm{N} ; 80^{\circ} 09.1^{\prime} \mathrm{W}\right)$

$\# 1 \mathrm{~A}(114), \# 1 \mathrm{~B}(99), \# 1 \mathrm{C}(96), \# 1 \mathrm{D}(91)$,

$\# 1 \mathrm{E}(122), \# 1 \mathrm{~F}(117)$,

-Site $2\left(25^{\circ} 25.3^{\prime} \mathrm{N} ; 8^{\circ} 09.1^{\prime} \mathrm{W}\right)$

\#"Old Yeller" core

-Site 3 (250 $21.7^{\prime} \mathrm{N} ; 8^{\circ} 10.2^{\prime} \mathrm{W}$ )

\#3A(102), \#3B(135),

-Site $4\left(25^{\circ} 23.9^{\prime} \mathrm{N} ; 8^{\circ} 10.2^{\prime} \mathrm{W}\right)$

\#4A(198), \#4B(140), 4C(119), \#4D(114),

\#4E(216), \#4F(185), \#4G(112)

-Site $5\left(25^{\circ} 36.8^{\prime} \mathrm{N} ; 80^{\circ} 06.1^{\prime} \mathrm{W}\right)$

$\# 5 \mathrm{~A}(71), \# 5 \mathrm{~B}(61)$

-September, 1986

-Site 5 (cont.)

$\# 5 \mathrm{C}(76)$, \#5D(48), \#5E(23), \#5F(51),

\#5G(63), \#5H(71), \#5I(74),

-Site $6\left(25^{\circ} 23.7^{\prime} \mathrm{N}\right.$; $\left.80^{\circ} 08.1^{\prime} \mathrm{W}\right)$ $\# 6 \mathrm{~A}(147), \# 6 \mathrm{~A}^{\prime}(185), \# 6 \mathrm{~B}(117)$

-Site $7\left(25^{\circ} 20.6^{\prime} \mathrm{N} ; 80^{\circ} 11.0^{\prime} \mathrm{W}\right)$

\#7A(99), \#7B(48), \#7C(117), \#7D(168), \#7E(114)

-Site 8 ( $25^{\circ} 35.3^{\prime} \mathrm{N} ; 80^{\circ} 09.2^{\prime} \mathrm{W}$ )

$\# 8 \mathrm{~B}(114), \# 8 \mathrm{C}(53), \# 8 \mathrm{E}(107), \# 8 \mathrm{~F}(48), \# 8 \mathrm{G}(122)$

-Elliott Key $\left(25^{\circ} 24^{\prime} \mathrm{N} ; 80^{\circ} 13^{\prime} \mathrm{W}\right)$

-September, 1977

-Cores

\#MKR(102), \#Mid-Elliott(94)

-Florida Bay $\left(24^{\circ} 53^{\prime} \mathrm{N} ; 80^{\circ} 45^{\prime} \mathrm{W}\right)$

-October, 1986

-Cores

\#5(102), \#5'(102), \#6(84) 
-Hen and Chickens Reef (240 56' $N$; $\left.80^{\circ} 33^{\prime} \mathrm{W}\right)$

-August, 1980

-Cores

$\# 1(79), \# 2(63), \# 3(119), \# 4(51), \# 5(130)$, $\# 6(109), \# 7(46), \# 8(112), \# 9(66), \# 10(56)$, \#13(165), \#14(63)

\section{-Looe Key Reef (240 33' N; $\left.81024^{\prime} W\right)$}

-August, 1980

-Cores

\#1(61), \#2(56), \#3(69), \#4(102), \#5(119), $\# 6(107), \# 7(84), \# 8(76), \# 9(76), \# 10(51)$, \#11(107), \#12(61)

-July, 1983

-Cores

$\# 1(99), \# 2(102), \# 3(102), \# 4(102), \# 5(61)$, $\# 6(84), \# 7(122)$, \#8(104)

-Short Cores

$\# 2(23), \# 2 '(18), \# 3(23), \# 4(23), \# 5(10)$, \#6(10), \#7(10), \#8(15), \#9(10), \#10(10), $\# 11(10), \# 12(25)$

-Bleaching Study, 1989

-Cores

\#Biggest Head (10 Pieces)(244)

-Random, Mixed Short Cores

\#1(25), \#2(36), \#3(25), \#4(36), \#5(25),

\#A-8(25), \#C-4(36), \#C-5(25), \#C-6(25),

\#D-2(28), \#E-4(25), \#F-6(25), G-7(25),

\#H-7(25), \#H-11(25), \#I-3(28),

\#HC-1(25), \#HC-2(25), \#HC-3(30),

\#Big Head(25), \#Twin Coral(25)

-Marquesas Keys $\left(24035^{\prime} N\right.$; $\left.82011^{\prime} \mathrm{W}\right)$

-August, 1980

-Cores

\#1(178), \#3(71), \#4(38), \#5(79), \#6(66)

-Ragged Keys (250 28' $N$; $80^{\circ}$ 10' W)

-March, 1975

-Core

-April, 1980

$\# 1(25)$

-Core

\#1(61) 
-New Ground Shoal (240 41' N; 82' 23' W

-July, 1983

-Cores

\#1(46), \#2(25)

-Davis Reef (240 56' $N$; $\left.82^{\circ} 30^{\prime} W\right)$

-September, 1974

-Core

\#1(33)

-Mosquito Bank (250 4'N; $\left.80^{\circ} 24^{\prime} W\right)$

-August, 1980

-Cores

\#1(43), \#2(61), \#3(43), \#4(43), \#5(43), \#6(43), \#7(43), \#8(41), \#9(43), \#10(36), \#11(30), \#12(53)

-Key Largo Marine Sanctuary

-August, 1978

-Site A Cores (250 15.7' N; $80^{\circ} 15.1^{\prime} \mathrm{W}$ )

\#A1(61), \#A2(91), \#A3(58), \#A4(46),

\#A5(58), \#A6(58), \#A7(58), \#A8(48),

\#A9(61), \#A10(46), \#A11(48), \#A12(58)

-Site B Cores (250 16.4' N; 80013.9' W)

\#B1(56), \#B2(114), \#B2-A(58), \#B3(58),

\#B4(56), \#B5(58), \#B6(58), \#B7(53),

\#B8(58), \#B9(56), \#B10(51), \#B11(58),

\#B12(22)

-Site C Cores (250 13.0' N; $80^{\circ} 12.9^{\prime} \mathrm{W}$ )

\#C1(119), \#C2(48), \#C3(58), \#C4(61),

\#C5(66), \#C6(56), \#C7(71), \#C8(56),

\#C9(56), \#C10(61), \#C11(53), \#C12(56)

-Site D Cores ( $25^{\circ} 13.5^{\prime} \mathrm{N} ; 80^{\circ} 16.4^{\prime} \mathrm{W}$ )

\#D1(58), \#D2(58), \#D3(53), \#D4(51),

\#D5(130), \#D6(58), D7(58), \#D8(56),

\#D9(56), \#D10(48), \#D11(56), \#D12(43) 
-Site E Cores ( $\left.25^{\circ} 13.1^{\prime} \mathrm{N} ; 80^{\circ} 15.7^{\prime} \mathrm{W}\right)$

\#E1(43), \#E2(58), \#E3(53), \#E4(51), \#E5(84), \#E6(48), \#E7(86), \#E8(56), \#E9(56), \#E10(48), \#E11(58), \#E12(56)

-Site F Cores ( $25^{\circ} 15.7^{\prime} \mathrm{N} ; 80^{\circ} 07.1^{\prime} \mathrm{W}$ )

$\# \mathrm{~F} 1$ (112), \#F2(53), \#F3(53), \#F4(56), \#F5(51), \#F6(61), \#F7(51), \#F8(56), \#F9(61), \#F10(117), \#F11(53), \#F12(58)

-Site G Cores ( $25^{\circ} 04.5^{\prime} \mathrm{N} ; 80^{\circ} 23.4^{\prime} \mathrm{W}$ )

\#G1(48), \#G2(51), \#G3(51), \#G4(53), \#G5(56), \#G6(58), \#G7(43), \#G8(53), \#G9(71), \#G10(58), \#G11(58), \#G12(41)

-Site $H$ Cores $\left(25^{\circ} 01.9^{\prime} \mathrm{N} ; 80^{\circ} 23.5 \mathrm{~W}\right)$

\#H1(43), \#H2(58), \#H3(58), \#H4(58), \#H5(58), \#H6(56), \#H7(53), \#H8(56), \#H9(53), \#H10(56), \#H11(86), \#H12(56)

-Site I Cores ( $25^{\circ} 03.0^{\prime} \mathrm{N} ; 80^{\circ} 21.3^{\prime} \mathrm{W}$ )

\#I1(58), \#I2(58), \#I3(56), \#I4(61), \#I5(56), \#I6(56), \#I7(58), \#I8(53), \#I9(53), \#I10(58), \#I11(53), \#I12(46)

-Site J Cores ( $\left.25^{\circ} 07.4^{\prime} \mathrm{N} ; 80^{\circ} 21.0^{\prime} \mathrm{W}\right)$

\#J1(53), \#J2(58), \#J3(56), \#J4(51), \#J5(94), \#J6(53), \#J7(51), \#J8(58), \#J9(51), \#J10(56), \#J11(56), \#J12(61)

-Site K Cores ( $\left.25^{\circ} 05.1^{\prime} \mathrm{N}, 80^{\circ} 18.4^{\prime} \mathrm{W}\right)$

\#K1(53), \#K2(56), \#K3(23), \#K4(53), \#K5(33), \#K6(56), \#K7(30), \#K8(28), \#K9(43), \#K10(53), \#K11(51), \#K12(76)

-Site L Cores ( $25^{\circ} 00.9^{\prime} \mathrm{N} ; 80^{\circ} 22.2^{\prime} \mathrm{W}$ )

\#L1(102), \#L2(53), \#L3(51), \#L4(43), \#L5(56), \#L6(48), \#L7(58), \#L8(58), \#L9(58), \#L10(71), \#L11(64), \#L12(48) 


\section{Florida Pleistocene (Key Largo LImestone)}

-Key Largo Cut $\left(250\right.$ 08' $\left.\mathrm{N} ; 80^{\circ} 24^{\prime} \mathrm{W}\right)$

$-1977$

-Slabs

$$
\text { \#1(66), \#2(64), \#3(30) }
$$

-Elliot Key (250 24' $\left.\mathrm{N} ; 80^{\circ} \mathrm{13}^{\prime} \mathrm{W}\right)$

$-1977$

- Slab

$\# 1(41)$

\section{Gulf of Mexico}

-East Flower Gardens $\left(27056^{\prime} N\right.$; $\left.93^{\circ} 36^{\prime} W\right)$

-August, 1979

-Cores

$\# 1(66), \# 2(66), \# 3(66), \# 4(61), \# 5(61)$, \#6(53), \#7(61), \#8(53), \#9(66), \#10(61), \#11(66), \#12(63), \#D. strigosa(36)

-West Flower Gardens (270 53' $\mathrm{N} ; 93^{\circ} 4^{\prime} 9^{\prime} \mathrm{W}$ )

-October, 1982

-Cores

\#1(35), \#2(35), \#3(33), \#4(35), \#5(35),

$\# 6(15), \# 7(20)$, \#8(35), \#9(34), \#10(33),

$\# 11(35), \# 2-1(34), \# 2-1 \mathrm{~A}(35), \# 2 \mathrm{~A}(35)$,

\#3A(36), \#4A(20), \#5A(35)

\section{Bahamas}

-Hogsty Reef $\left(21^{\circ} 42^{\prime} \mathrm{N} ; 7^{\circ} 4^{\circ} 47^{\prime} \mathrm{W}\right)$

-December, 1978

-Cores

$\# 1(66), \# 2(64), \# 3(127), \# 4(114), \# 5(112)$,

$\# 6(69)$ 


\section{Bellze}

-Carrie Bow Key $\left(16^{\circ} 48^{\prime} \mathrm{N} ; 88^{\circ}\right.$ 05' W)

-July and August, 1977

-Cores

$$
\# 1(102), \# 2(28), \# 3(28), \# 4(48)
$$

-Glover's Reef (16045' N;87045 W)

-July, 1977

-Cores

$\# \mathrm{~A}(31), \# \mathrm{~A}^{\prime}(31), \# \mathrm{~B}(30), \# \mathrm{C}(33), \# \mathrm{D}(64)$

\section{Puerto Rico}

-Turrumate I Cay (170 56' N;660 58' W)

-July, 1982

-Core

\#2(119), \#10(66)

-Cayo Enrique (170 57' $N$; 670 03' W)

-July, 1982

-Core

$\# 1(102)$

-Rodriguez Reef (180 10' N;670 11' W)

-July, 1982

-Core

\#1(61), \#2(94) 


\title{
Virgln Islands
}

-St. Croix $\left(17045^{\prime} \mathrm{N} ; 64^{\circ} 35^{\prime \prime} \mathrm{W}\right)$

-December, 1976

-Cores

\#1 (Dendrogyra cylindrus)(20), \#2(31), \#3(27), \#4(29), \#5(25), \#6(30), \#7(31), \#8(28),

\#9(20), \#10(30), \#11(28), \#12(28),

\#13(30), \#14(27), \#15(31), \#16(31),

\#17(22), \#18(30), \#19(33)

\section{Marshall Islands}

-Enewetak Atoll (Porites lutea) $\left(11^{\circ} 40^{\prime} \mathrm{N} ; 162^{\circ} 15^{\prime} \mathrm{E}\right)$

-July, 1984

-Cores

$$
\begin{aligned}
& \# 1(69), \# 2(36), \# 3(28), \# 4(25), \# 5(28), \\
& \# 6(36), \# 7(30), \# 8(74), \# 9(244), \# 10(150), \\
& \# 11(173), \# 13(180), \# 14(135), \# 15(262)
\end{aligned}
$$

\section{Philippine Islands}

-Nido Field (off Palawan Island) (110 29' N; 1190 01" E)

-May, 1981

-Core

\author{
\#1(117)(Porites lobata)
}

